\title{
Discussion of talks from the symposium: Factor X: From thrombokinase to oral anti-coagulants and beyond
}

\author{
Leonard M. Milstone ${ }^{1} \cdot$ Craig Jackson $^{2} \cdot$ Richard C. Becker $^{3} \cdot$ Rodney Camire ${ }^{4} \cdot$ Robert Knabb $^{5} \cdot$ Kenneth Mann $^{6}$. \\ Wolfram Ruf $^{7,8} \cdot$ Ruth R. Wexler $^{5}$. Jeremy Wood ${ }^{9}$
}

Accepted: 23 July 2021 / Published online: 12 October 2021

(c) The Author(s), under exclusive licence to Springer Science+Business Media, LLC, part of Springer Nature 2021

\section{Milstone talk discussion}

The article, "Factor Xa: Thrombokinase from Paul Morawitz to J Haskell Milstone" by Leonard M. Milstone can be found here: https://link.springer.com/article/10.1007\%2Fs11 239-021-02387-6

Craig Jackson: Do you have any insights into why Haskell Milstone's observations were largely overlooked?

Leonard Milstone: Well, the simple answer to that he was reserved and humble. But it's obviously a quite a bit more complicated than that. He had a comprehensive command of the literature and a strong sense of historical perspective; he always put his work in context with what others had done or were doing. This was a habit not common to the field at the time. He had very high standards for accuracy in speech and writing, and I think he was painfully aware that

Leonard M. Milstone

leonard.milstone@yale.edu

Craig Jackson

CraigMJacksonSD@outlook.com

Richard C. Becker

Richard.becker@uc.edu; BECKERRC@ucmail.uc.edu

Rodney Camire

rcamire@ pennmedicine.upenn.edu

Robert Knabb

rmk1955@gmail.com

Kenneth Mann

kgmann1@comcast.net

Wolfram Ruf

ruf@scripps.edu; ruf@uni-mainz.de

Ruth R. Wexler

ruth.wexler@bms.com

Jeremy Wood

jeremy.wood@uky.edu

1 Department of Dermatology, Yale School of Medicine, New Haven, CT 06510-2059, USA the technology at hand was DOCA inadequate to answer questions about the relationship of his thrombokinase to other coagulation factors. He also wasn't a self-promoter. He rarely went to meetings. He enjoyed working alone. His small laboratory did not graduate many acolytes who talked about him or his work after they left his laboratory. He published a total of 38 papers; 35 about coagulation. Many contemporaries working in coagulation published hundreds.

Jeremy Wood: Is it possible that Morawitz had actually identified factor $\mathrm{V}$ in platelets when he proposed that platelets were contributing to thrombokinase?

Milstone: Factor V must have been present in his assays. To me, and I think to Haskell Milstone, Morawitz's unique contribution was the idea that an enzyme activated prothrombin. Morawitz's idea that thrombokinase might come from platelet extracts fit well with the prevailing idea that

2 San Diego, USA

3 University of Cincinnati College of Medicine, 231 Albert Sabin Way, Cincinnati, OH 45267, USA

4 University of Pennsylvania, The Colket Translational Research Bldg., Room 5018, 3501 Civic Center Blvd., Philadelphia, PA 19104-4399, USA

5 Bristol Myers Squibb Company, P.O. Box 5400, Princeton, NJ 08543, USA

6 Department of Biochemistry, Larner College of Medicine, University of Vermont, C401 Given Building, 89 Beaumont Avenue, Burlington, VT 05405, USA

7 The Scripps Research Inst, 10550 N Torrey Pines Rd, SP280, La Jolla, CA 92037-1000, USA

8 Center for Thrombosis and Hemostasis (CTH), University Medical Center, Johannes Gutenberg-University Mainz, Mainz, Germany

9 Cardiovascular Research Center, University of Kentucky, Lexington, KY 40536-0298, USA 
cells initiated coagulated. To this day, many overemphasize the cellular origin of Morawitz's thrombokinase-even conflating it with Tissue Factor or Factor V-and underemphasize Morawitz's foresighted belief that thrombokinase was an enzyme.

Rodney Camire: How did Owren's description of Factor $\mathrm{V}$ impact your father's thoughts on the function of thrombokinase?

Milstone: He quoted Owren's work many times, and in his 1952 review struggled to fit Owren's factor V into his own coagulation scheme. By contrast, Owren originally believed that his Factor $\mathrm{V}$ was the proenzyme that activated prothrombin and that thrombokinase, a term he used interchangeably with thromboplastin, was in the brain extract that facilitated Factor V action. The existence of Milstone's plasma-derived thrombokinase never seemed to have troubled him. I don't believe that they ever exchanged reagents.

\section{Jackson talk discussion}

The article: "Structure and function of factor X: properties, activation, and activity in prothrombinase. A retrospective in a historical context" by Craig M. Jackson can be found here: https://link.springer.com/article/10.1007/ s11239-021-02421-7

Camire: What was the thinking about differences in function of the two different forms of factor $\mathrm{X}$ that you isolated?

Jackson: I had found no evidence for differences in activation rate. Takashi Morita came to the lab and did a superb job of job of showing that sulfation of tyrosine in the activation peptide distinguished the two forms. No functional differences were found.

Camire: Were there concurrent thoughts about functional impact of the alpha and beta forms that appear following conversion of $\mathrm{X}$ to $\mathrm{Xa}$ ?

Jackson: Well, there was much of a thought process. I saw no differences in Xa activity, but this was the last thing I did before beginning to write my dissertation; very limited experiments.

Milstone: Craig, SDS gels were clearly an important part of your work. What were you expecting to learn from the different fragments on the gels?

Jackson: Whyte Owen came to the lab to identify the function of the pro piece of prothrombin. The SDS gels, first the Weber-Osborne then the Laemmli systems, were important in that work because you didn't need much material to do a lot of experiments and they were independent of clotting experiments. They played an important role in rejecting Walter Seeger's view that alternate cleavage sites produced the other vitamin $\mathrm{K}$ dependent proteins from prothrombin.

Kenneth Mann: Thank you, Craig for bringing back many memories. I started out in physical chemistry and got into coagulation quite by accident. The field of coagulation was, shall we say, highly antagonistic. I have minutes from a Josiah Macy conference where Armand Quick and Walter Seegers were arguing about the merits of Quick's one-stage and Seegers' two-stage assays. At one point Quick says to Seegers, "you can best serve the field of coagulation by volunteering your brain for thromboplastin."

Jackson: Well, Ken, I started out in a lipid lab. Although you and I started with a different technological framework when we were graduate students, the conceptual framework was pretty clear: unique sequences of proteins were no longer doubted and proteolytic enzymes were, pretty much, considered to be what they are today. We were there in the right place at the right time. It was a fun time. We had the technologies; we had enough material to work with; so we could do experiments that were not imaginable previously. And, the lab was the focal point of our lives. Chuck Esmon and Naomi, then his girlfriend, and Whyte Owen and his wife would play bridge late into the night in the prep room because no one wanted to risk a fraction collector failing at a late stage in a purification.

\section{Mann talk discussion}

The article: "Prothrombinase: the paradigm for membrane bound enzyme complexes; a memoir" by Kenneth G. Mann can be found here: https://link.springer.com/article/10.1007/ s11239-021-02402-w

Milstone: Ken, you made extensive use of antibodies in your studies. Can you tell us how you came to use antibodies and how that changed the way you approached your work?

Mann: Well, the National Science Foundation once identified me as a creative scientist. I responded and said, I wasn't creative, I was adaptive. When I saw the Kohler-Milstein paper, I became immediately fascinated with the idea of monoclonal antibodies as reagents. I had a colleague, Jerry Katzmann, in the hematology division who knew what a mouse spleen looked like and I told him I wanted to make monoclonal antibodies to everything. The first antibody we made was to human Factor V which we coupled to Sepharose, and that allowed rapid purification of this otherwise labile factor from plasma. We developed a whole library of all the monoclonal antibodies to everything that we knew. One with anticoagulant activity was developed in conjunction with SmithKline Beecham. When SmithKline Beecham was acquired by Glaxo, they dumped the project because the CEO of Glaxo Smith Kline said; well, you have to realize that monoclonal antibodies will never be used for pharmacologic applications. 


\section{Ruf talk discussion}

The article "Roles of factor Xa beyond coagulation" by Wolfram Ruff can be found here: https://link.springer.com/ article/10.1007/s11239-021-02458-8

Milstone: I have a three-part question: First, when Xa is induced on monocytes are the carboxylase enzymes also induced? Second, have you seen Xa induction on cells other than monocytes? Third, in the tumor micro environment, in the absence of lipopolysaccharide, what is it that induces this series of enzymes?

Wolfram Ruf: In macrophages, in vitro, the activity of Factor Xa appearing in the supernatant is blocked by vitamin $\mathrm{K}$ antagonists. The full gamma carboxylase machinery is expressed both for the Factor VIIa and for the Factor Xa in macrophages and in immune cells. The list of responsive cells is growing, but the best data right now are for monocytes and macrophages. The endogenous signal in the tumor microenvironment is an open question. What do we know is that circulating monocytes make Factor $\mathrm{X}$ in tumor bearing mice, but we don't know the signal.

\section{Panel discussion}

Milstone: Craig and Ken: you started before protein sequencing was readily available, and institutions didn't provide it as a service. How easy or difficult it was to get sequencing done?

Mann: The Edman degradation method of sequencing was extraordinarily labor intensive. Even more importantly, it was extraordinarily material intensive.

Jackson: Getting amino acid analyses done when I was a graduate student was a strange exercise. The two amino acid analyzers at University of Washington were controlled by Hans Neurath. [Ken Mann laughs]. There was a political price to pay to get amino acid analysis. Fortunately, Dick Granberg, the tech who operated the analyzers, took pity on graduate students. I would take hydrolysates, stick the tubes in a drawer in the analysis lab, and Dick would run them overnight because Hans never came in at night. One or two mornings later, he would put the strip chart recordings with the peaks that had to be integrated in the same drawer where I could go early in the morning and pick them up before Hans would ever be there. It was more a political challenge than it was a technical challenge at that stage.

Mann: A lot of technology was under the control of, shall we say, fiefdoms. Access to an ultracentrifuge, an amino acid analyzer or a fluorometer would involve a genuflection, a kissing of the ring and various other behaviors. When I was recruited to the Mayo clinic in 1972, one serendipitous thing was that they basically said what do you need? And I said, everything. And they said anything else?

Jackson: I think there is incontrovertible evidence that I failed at kissing the ring. Roy Vagelos, Biochemistry Chair at University of Washington, had insisted upon reading all of the papers of junior faculty, so he was surprised when he received a letter panning everything I had done. He called me into his office and said, "You're stepping on the toes of some powerful people and you should be very careful about how you say things." Before Roy left to go to Merck he came to me and said, "I thought, I should tell you who sent the letter; it was Hans Neurath."

Jackson: Am I being a paradigm assassin in asking whether the division of hemostasis into an extrinsic and intrinsic systems is dead?

Ruf: Once you go beyond initiation, you must think about interactions and amplification loops. You have new thinking about platelet localized amplification of Factor XI activation and inflammation and the thrombin feedback loop. So I think we need to better understand the relative importance under physiological and pathological conditions of flow or vascular diseases. I think that's where the field is moving and there's a lot to be done still.

Mann: With respect to the intrinsic pathway as it has been considered in the past, Factor XI deficient patients do not bleed, and when was the last time you infused Factor XI or Factor XII into a deficient patient? The answer is: you don't because they don't need it for coagulation. So you do have to hinge everything on human physiology in the long run.

Jackson: Perhaps the contact system is an artifact of some of the earliest studies of coagulation, going back into the 1800s. It took decades to recognize that shed blood collected into a basin clotted because of the basin, not because anything else that was particularly significant. They did beautiful experiments, like tying off blood vessels and noting how long the blood would remain fluid under those circumstances-which again goes back to the endothelial protective role for blood vessels.

Milstone: Up until the 1970s if you wanted to ascribe a particular activity to a protein, you had to find a family that lacked it or you had to prove that your isolated protein was pure. How has recombinant technology changed that approach?

Mann: With recombinant technology you can go the full circle from the isolated, natural material to the synthesis of that material de novo to prove that it's the same material. One of the risks of the in vitro synthesis of proteins for activity studies is the absence of appropriate post-translational modifications, such as glycosylation and gamma carboxylation.

Ruf: But with recombinant technology, especially now with CRISPR, we can bring the biochemistry back into the mouse and get a naturally modified protein in a complete 
biological system. Then by introducing single point mutations in mice, such as the ones I described, we can really come to very definitive answers on mechanism.

\section{Camire talk discussion}

The article "Blood coagulation factor X: molecular biology, inherited disease, and engineered therapeutics" by Rodney M. Camire can be found here: https://link.springer.com/artic le/10.1007/s11239-021-02456-w

Milstone: Do antibodies develop to these engineered variants and would they limit repeated or long-term use?

Camire: No neutralizing antibodies were detected in the clinical trials. In the animal studies that we've done none were seen. In trials of other engineered proteins for hemophilia, some neutralizing antibodies appeared in phase three. So, I suspect that since the Xa variant is a mutant, it is possible that neutralizing antibodies might appear after repeated dosing.

Milstone: How did your move to Katherine High's lab impact the direction of your work?

Camire: I did my graduate work with Paula Tracy at the University of Vermont in a really rich environment for coagulation factor biochemistry. When I went to UPENN for a post-doc in Kathy's lab, I never really thought I would be getting into in vivo assessment of coagulation proteins. But Kathy's lab was deeply involved in gene therapy. I was probably the only person in her lab not doing that. But that environment exposed me to the thought process of testing new molecules in dog and mouse models of hemophilia. So that had a huge impact on the direction I took when I started my own lab.

Ruf: How do you judge the opportunities for using zymogen-like mutants for gene therapy? Would it allow you to adjust the expression levels and improve safety, for example for hemophilia A?

Camire: There are two different approaches here that one could take. You could express the zymogen mutants as Factor $\mathrm{X}$ or further engineer the variants so they are expressed as "active" zymogen-like variants without the activation peptide. We did some preliminary work on this second approach and found that expressing FXa-I16L led to signs of thrombosis. This is probably not too surprising as a little bit of Xa activity for a long period of time is not good. The animals did not do very well; you don't need a lot of $X$ a to cause a lot of havoc in terms of thrombin generation. With this approach, it is difficult to try to find the right dosage that could be effective. We never really pushed it too far beyond these preliminary experiments. In my opinion, continuous expression in a gene therapy setting of low-level activity of Xa is probably not the best way to go. Maybe we didn't have the right variant as the activity of FXa-I16L is probably a little too high, or it's not zymogen-like enough for this approach.

\section{Knabb-Wexler talk discussion}

The article "From basic science to life-saving therapy: the rationale, and drug discovery efforts that led to the direct factor Xa inhibitor eliquis" by Robert M. Knabb and Ruth R. Wexler can be found here: https://link.springer.com/artic le/10.1007/s11239-021-02529-w

Milstone: This is a staggering amount of work, but you have to be pleased with the outcome. Ruth mentioned that there were close to 30 chemists and 7000 or 10,000 compounds that were tested. Robert, can you give us some idea of the number of people and studies that were needed to advance these compounds through efficacy, in vivo toxicity, PK and final dosing?

Robert Knabb: While chemists were pretty much dedicated to a program at any given time, it's harder to quantify the number of biologists, PK scientists and others who worked on the program because we utilized resources of a lot of different groups that simultaneously worked on several projects. The work that was done to characterize the PK we did ourselves, but there was a separate analytical group to analyze the samples. You can think of drug development as a large funnel and at each step the funnel narrows. Screening of 10,000 compounds for Factor Xa inhibition and selectivity was a high throughput assay in microtiter plates. A small fraction of those with appropriate potency and selectivity, perhaps one in ten, were evaluated in a plasma based clotting assay. Then, the best ones go into in vitro PK assays. I would say that we probably took $50-100$ compounds into the animal efficacy models and 40 or 50 compounds for PK in the chimpanzees. Then we also took about seven compounds into development, of which five went into phase one clinical trials. At each step of the way the team gets larger and larger, but you're utilizing the resources that are divided amongst many different therapeutic programs.

Ruth Wexler: I think it is important to note that if you have strong belief that a target has the potential to be transformational, you want to ensure that you have selected the right compound, the highest quality compound that can go into full development. To clarify one point, the numbers 7000 and 10,000 are both correct for our Factor Xa program. Apixaban was approximately the seven thousandth compound synthesized in the program, and we had a backup program which continued after apixaban was synthesized, so in total approximately 10,000 compounds were synthesized. In fact, we took two compounds after apixaban into phase one studies because we wanted to be sure that we had a compound that would go all the way to becoming a new medicine. 
Milstone: Prior to developing DOCAs did DuPont have an existing program for serine protease inhibitors?

Knabb: DuPont sold Coumadin, which they had acquired that from Endo Pharmaceuticals. At DuPont, we had a real interest in discovering and developing a successor for Coumadin, so we worked on both anticoagulants and antiplatelet agents. We conducted research and chemistry on both thrombin and Factor Xa inhibitors (the thrombin inhibitor program started first with boronic acid inhibitors). Work from our own labs, as well as emerging studies from academia, suggested a preferable profile of Xa inhibitors over thrombin inhibitors.

Wexler: Yes, in the early nineties we were running a thrombin inhibitor program, but weren't yet working on Factor Xa. When we became DuPont Merck, our head of research came from Merck and he was interested in Factor Xa. As Bob said we started a Factor Xa program, subsequently based on our own work as well as emerging science from academia. We had strong reasons to believe in Factor $\mathrm{Xa}$ as the preferable target and then focused solely on Factor Xa.

Jackson: The S4 site seemed to be the key site for achieving your objectives. Once crystallography became available how important was it for identifying the properties that you desired?

Wexler: Yes, the S4 subsite was important in the final optimization culminating in apixaban. This optimization at the S4 site was to achieve the desired pharmacokinetic properties while maintaining excellent potency and selectivity and was accomplished by identifying low clearance, low volume of distribution compounds. This was achieved by adjusting the physicochemical properties of the molecules by utilizing a terminal neutral moiety, such as the lactam as the P4 moiety which resulted in apixaban. Crystallography and structure-based drug design were important for optimizing potency and selectivity largely in other parts of the molecule.

Ruf: Some of my interest has been in the function of $\mathrm{Xa}$ inhibitors in the extravascular space. As you developed apixaban, what information did you develop on bio-distribution of these compounds in extra vascular tissues? Was there an effort to avoid the compound getting into extravascular space?

Knabb: It's really a complex problem. I don't think we ever specifically measured extracellular concentrations. The volume of distribution of apixaban is small compared to many drugs. But it's not strictly in the blood. Some of our earlier compounds caused intracellular cytotoxic effects. The larger percentage you can have in the blood, the more, your chances are for efficacy and the lower your chances are for untoward effects.

Milstone: Wolfram, does apixaban have the same anticancer effect his rivaroxaban?
Ruf: It's still an open question, but that's why I asked about biodistribution. With rivoraxaban we had striking differences between low molecular weight heparins, that are small enough in principle to go to the paravascular space, but are limited by where the antithrombin goes.

\section{Becker talk discussion}

The article "Factor Xa inhibitors: critical considerations for clinical development and testing" by Richard C. Becker can be found here: https://link.springer.com/article/10.1007\% 2Fs11239-021-02455-x

Milstone: Can you elaborate about why you were so careful in your presentation to specify prospective testing for each anti-coagulation indication and each individual valve?

Richard Becker: There is something very unique about valves in terms of contact activation, velocity of flow, and patients with concomitant atrial fibrillation. Early experience with dabigatran in patients with mechanical heart valves did not end as one would have hoped. In fact, there was a higher likelihood of bleeding and a higher likelihood of valve thrombosis and thromboembolic events. The Onyx valve is still a pyrolytic carbon valve, but has geometry and flow characteristics that are unique and we knew that thrombosis was less likely for valves in the aortic position. That being said we were still venturing into territory that is to some degree, uncharted. Aware of the need for caution, we began a 2-year conversation with the FDA that resulted in gathering additional data; making sure that our data and safety monitoring committee was experienced and in place; that the design would be an open label trial for the first 3 months after valve insertion, and that patients would be treated with warfarin. We felt that that the first 3 months would be the highest risk period, but these individuals would require lifelong anticoagulation therapy. So why not look carefully at fixed dosing for someone that is going to require treatment for 2 years, 5 years, 10 years, 20 years.

Ruf: Richard, could you comment on what you might expect from using oral Xa inhibitors in the thrombo-inflammatory aspects of COVID-19? Would they simply target thrombosis or also target some immune pathways? Could there be some adverse outcome, such as an effect of antiviral immunity?

Becker: I would welcome your perspective, too. We have pushed a very strong agenda for the NIH ACTIV-4 trials, which explore the use of antithrombotic therapy, antiplatelet therapy, anticoagulant therapy, in COVID-19. This would include its use in combination with remdesivir, convalescent plasma and other therapies that are now becoming standards of care. Will these lead to reduced mortality and, if so, will that reduction in mortality be thrombosis driven at the 
microvascular level at the macrovascular level? These are unknown. So what do you think?

Ruf: I'm kind of torn. I still have the anti-phospholipid trial in my mind from a couple of years ago. There was great hope for Xa inhibitors in severe anti-phospholipid syndrome. The results were scary and the trial was interrupted. There was an enormous incidence of arterial thrombosis, which is part of the syndrome, in spite of standard-of-care anti-coagulation. Nobody has a good explanation yet. In the context of COVID you could have beneficial or adverse effects. I would not put a bet out either way right now.

\section{Panel discussion}

Milstone: I have a different kind of question for the afternoon speakers. Most academics have laboratories with 10 or maybe 15 people in them at one time. Drug development and testing involves hundreds. What is your secret for managing such a large team?

Wexler: A lot of it is a shared passion for the science and for the notion that, if and when we're successful, we're going to make a huge difference in the lives of patients. The other thing that's really important is persistence. There's a lot of failure in drug discovery, you just to recognize that it comes with the territory. It's okay to fail; lessons learned from experiments that don't work are as important as those that do. You just have to get back up every time you fail. And don't underestimate teamwork. Every member of the team has an important role to play, so making people feel valued is really critical.

Mann: My career was based upon having bright people walk through the door, simple as that. Michael Nesheim, the guy that isolated Factor V and basically defined the kinetics and assembly of prothrombinase, came to my laboratory because he was fired by his preceptor, because he was too independent.

Becker: I'll make a brief comment about the clinical research science side of things. The one thing that became very clear to me early in my career is that there's good science and there's bad science. Very large groups and many clinical sites have to maintain the same level of rigor as the individual working at the bench. The scale may seem different, but the scientific methods that we hold dear are what allows good science to be done in drug development.

Knabb: I had the great privilege of being both part of the discovery and clinical development and registration of Eliquis. Partnerships between the pharmaceutical industry and academic medicine were fulfilling and productive and really allowed us to conduct trials that were of the highest quality. Understanding that collaboration with regulatory agencies, who watch over these things very carefully, is often an iterative process where you both seek the best design that will give the critical answers to whether this therapeutic will be an improved therapy in this group of patients. It's being open to those kinds of collaborations that makes dealing with large groups work.

Milstone: Let me conclude by thanking you all for sitting through this long day. Early workers may have thought that thrombokinase/Factor X would be just another serine protease. What we've learned today is that the regulation of its activities and it's molecular interactions are very complicated, and there is still much to learn.

Publisher's Note Springer Nature remains neutral with regard to jurisdictional claims in published maps and institutional affiliations. 